\title{
Nanoscale
}

Check for updates

Cite this: Nanoscale, 2019, 11, 4190

Received 9th November 2018,

Accepted 9th January 2019

DOI: $10.1039 / c 8 n r 09042 d$

rsc.li/nanoscale

\section{Silicon substrate effects on ionic current blockade in solid-state nanopores $\uparrow$}

\author{
Makusu Tsutsui, (iD *a Kazumichi Yokota, ${ }^{a, b}$ Tomoko Nakada, ${ }^{a}$ Akihide Arima, (iD a \\ Wataru Tonomura, ${ }^{a}$ Masateru Taniguchi, ${ }^{a}$ Takashi Washio ${ }^{* a}$ and Tomoji Kawai*a
}

\begin{abstract}
We investigated the roles of silicon substrate material compositions in ionic current blockade in solid-state nanopores. When detecting single nanoparticles using an ionic current in a $\mathrm{Si}_{3} \mathrm{~N}_{4}$ nanopore supported on a doped silicon wafer, resistive pulses were found to be blunted significantly via signal retardation due to predominant contributions of large capacitance at the ultrathin membrane. Unexpectedly, in contrast, changing the substrate material to nondoped silicon led to the sharpening of the spike-like signal feature, suggesting a better temporal resolution of the cross-channel ionic current measurements by virtue of the thick intrinsic semiconductor layer that served to diminish the net chip capacitance. The present results suggest the importance of the choice of $\mathrm{Si}$ compositions regarding the capacitance effects to attain better spatiotemporal resolution in solid-state nanopore sensors.
\end{abstract}

\section{Introduction}

Solid-state nanopores have been widely used as a useful sensor platform for analyzing single particles and single molecules in liquid. ${ }^{1-5}$ The detection principle involves measurements of an ionic current through a nanoscale hole sculpted in a thin dielectric membrane supported usually on a Si wafer. ${ }^{6}$ This simple mechanism allows one to detect an object passing through the conduit as it excludes ions there, thereby causing a pulse-like decrease in the current. The thus recorded resistive pulse contains valuable information concerning multiple physical parameters of single particles, including volume, ${ }^{7,8}$ shape,${ }^{9,10}$ mass, ${ }^{11}$ surface charges ${ }^{12}$ and even surface proteins. ${ }^{13}$

Similar to any sensor devices, a signal-to-noise ratio is a crucial issue in nanopore sensing. Usually, this has been dealt

\footnotetext{
${ }^{a}$ The Institute of Scientific and Industrial Research, Osaka University, Japan. E-mail: tsutsui@sanken.osaka-u.ac.jp,washio@ar.sanken.osaka-u.ac.jp, kawai@sanken.osaka-u.ac.jp

${ }^{b}$ National Institute of Advanced Industrial Science and Technology, Japan $\dagger$ Electronic supplementary information (ESI) available. See DOI: 10.1039/ c8nr09042d
}

with by designing the channel size closer to the analytes of concern so as to produce more pronounced ionic current spikes by letting a larger amount of in-pore ion blockage upon translocation relative to the channel volume. ${ }^{7}$ Surface coating has also proven to be promising for suppressing the noise stemming from electrochemical reactions at water-dielectric interfaces ${ }^{14}$ as well as for coupling of amplifier voltage noise to device capacitance. ${ }^{15-18}$ In addition to these strategies, the choice of substrate materials has been found to be a more basic yet effective way for improving the sensitivity of singleparticle detectors. ${ }^{19,20}$ For instance, compared to widely employed $\mathrm{Si}$, low- $k$ materials such as glass and polymers were demonstrated to offer a lower noise platform. ${ }^{19}$ However, non$\mathrm{Si}$ substrates are not compatible in general with semiconductor technologies due to the rough surface that necessitates special skills to form membranes and pores. ${ }^{19}$ In contrast, we herein show that intrinsic Si can still be useful as a nanopore substrate regarding the spatiotemporal resolution of the ionic current-based single-particle analyses.

\section{Results and discussion}

\section{Ionic current in a nanopore on doped $\mathrm{Si}$}

While the good insulating property of intrinsic Si posed a difficulty for exploiting electron-beam lithography to delineate nanopores, we developed a process to finely define a nanoscale channel by inserting a conductive thin film on a resist layer. By this process, we fabricated a pore of diameter $d_{\text {pore }}$ in a $50 \mathrm{~nm}$ thick $\mathrm{Si}_{3} \mathrm{~N}_{4}$ membrane suspended on doped (1-20 $\Omega \mathrm{cm}$ ) or non-doped (>1000 $\Omega \mathrm{cm}) \mathrm{Si}$ substrates and used it for the resistive pulse analyses of carboxylated polystyrene nanoparticles of diameter $d_{\mathrm{PS}}$ in an electrolyte solution.

The cross-membrane ionic current $I_{\text {ion }}$ versus time $(t)$ curves in $1 \times$ PBS with a nanopore of $d_{\text {pore }}=300 \mathrm{~nm}$ supported on a doped Si substrate (Fig. 1, see also Fig. S1 $\dagger$ ) showed an open pore current of $61 \mathrm{nA}$. This ionic conductance is in quantitative accordance withthe analytical expression ${ }^{21,22}$ of the ionic 
a

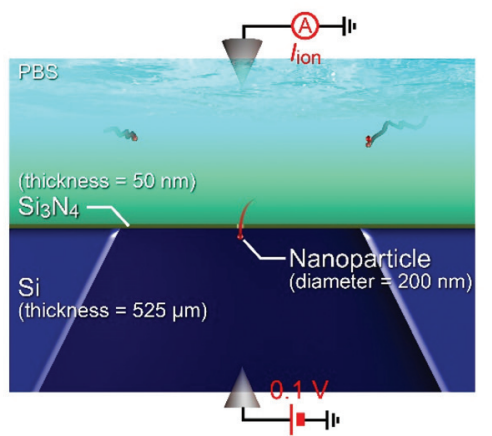

b

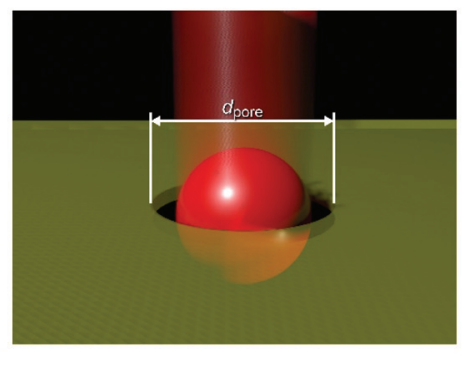

C

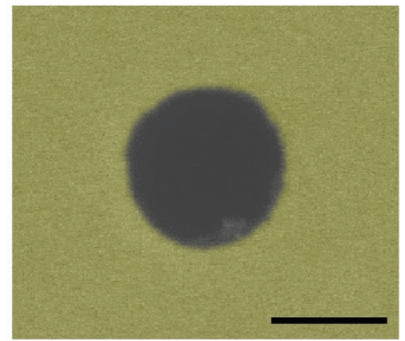

Fig. 1 Solid-state nanopore structure. ( $a$ and b) Schematic models depicting single-nanoparticle detection in an electrolyte solution using a solidstate nanopore supported on a Si substrate. Two $\mathrm{Ag} / \mathrm{AgCl}$ electrodes were utilized to apply a dc voltage of $0.1 \mathrm{~V}$ to a pore of diameter $d_{\text {pore }}$ (b) in a thin membrane and measure the ionic current $I_{\text {ion }}$ flowing through therein. (c) False-colored scanning electron micrograph of a nanopore of $d_{\text {pore }}=$ $300 \mathrm{~nm}$ sculpted in a $50 \mathrm{~nm}$ thick $\mathrm{Si}_{3} \mathrm{~N}_{4}$ membrane. Scale bar denotes $200 \mathrm{~nm}$.

resistance $R_{\text {pore }}$ inside the channel, $R_{\text {pore }}=\rho\left(4 t_{\text {pore }} / \pi d_{\text {pore }}^{2}+\right.$ $\left.1 / d_{\text {pore }}\right)=1.6 \mathrm{M} \Omega$ with the solution resistivity $\rho=0.4 \Omega \mathrm{m}$ for $1 \times$ PBS and the pore depth $t_{\text {pore }}=50 \mathrm{~nm}$ predicting $I_{\text {ion }}=62 \mathrm{nA}$ at $0.1 \mathrm{~V}$. On the other hand, resistive pulses were observed when admitting the buffer containing carboxylated polystyrene beads of $d_{\mathrm{PS}}=200 \mathrm{~nm}$, which are indicative of the electrophoretic translocation of the negatively charged polymeric nanoparticles through the channel (Fig. 2a). ${ }^{6}$ A close view of an $I_{\text {ion }}$ signal revealed a rather smooth change in the current reflect- ing the dynamic motions of the polymer sphere in the expansive sensing zone extending by more than $500 \mathrm{~nm}$ from the channel due to the ultra-low thickness-to-diameter aspect ratio motif of the $\mathrm{Si}_{3} \mathrm{~N}_{4}$ nanopore used (Fig. $2 \mathrm{~b}$ and c). ${ }^{23}$

\section{Anomalous cross-pore ionic current characteristics on non- doped $\mathrm{Si}$}

Unexpectedly, $I_{\text {ion }}$ traces of a nanopore on non-doped Si were found to be quite different. First of all, although the experi-

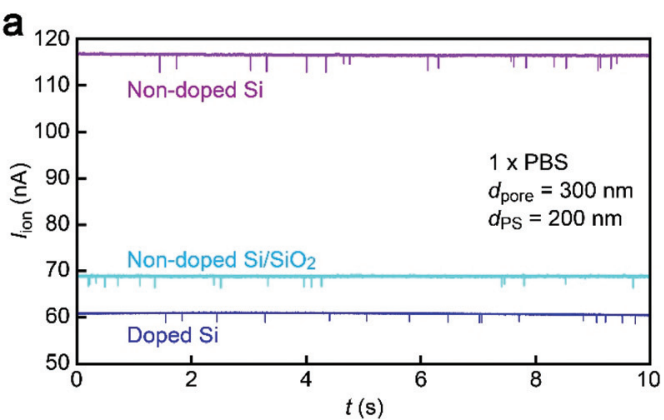

C

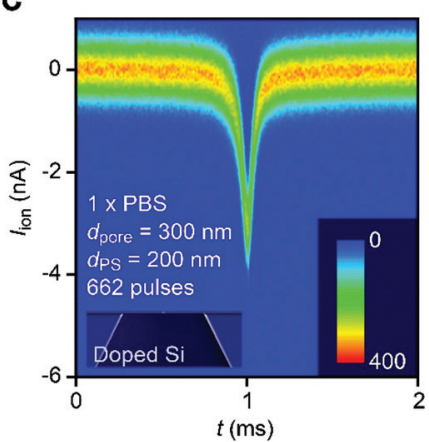

d

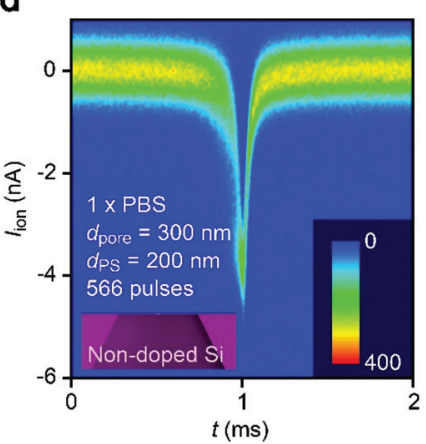

b

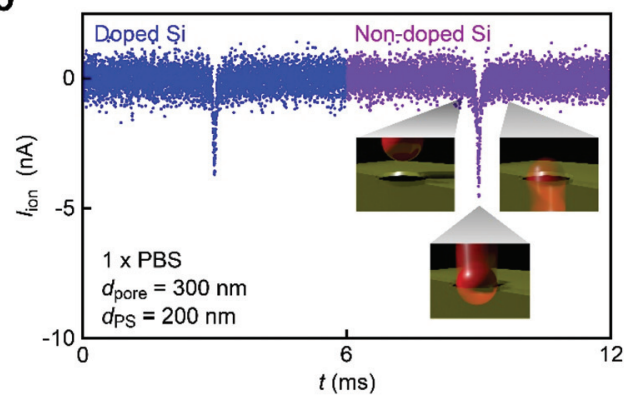

e

Fig. 2 Single-nanoparticle detection using a nanopore on a Si support of various compositions. (a) Temporal change in the ionic current ( $I_{\text {ion }}$ ) through a $300 \mathrm{~nm}$ sized nanopore supported on a substrate made up of doped Si (blue), non-doped Si (purple), or $\mathrm{SiO}_{2}$-coated non-doped Si (sky blue) in a dispersion solution of $200 \mathrm{~nm}$ sized carboxylated polystyrene nanoparticles in 1X PBS. Pulse-like signals indicate the electrophoretic translocation of the nanoparticles through the nanopore. (b) Magnified views of resistive pulses. The open pore current is offset to zero. Color coding is the same as that in (a). (c-e) Two-dimensional histograms showing a number of resistive pulses obtained with a $300 \mathrm{~nm}$ sized nanopore supported on a substrate of doped $\mathrm{Si}$ (c), non-doped $\mathrm{Si}$ (d), and $\mathrm{SiO}_{2}$-coated non-doped $\mathrm{Si}$ (e). 
mental conditions including ion concentrations and pore geometries were all set to be the same, the open pore current was higher by a factor of 2 than that in doped Si (Fig. 2a). Moreover, the ionic spike signals were also found to be slightly larger (Fig. 2b-d). This sort of difference cannot be simply ascribed to a variation in the pore diameter as larger pores should yield smaller resistive pulses in the low-thickness-todiameter aspect ratio pore structure, ${ }^{23}$ which is opposite to the case in Fig. 2b. In fact, the pore diameter was confirmed to differ by less than $10 \%$ under a scanning electron microscope (Fig. S1†). To shed light on this intriguing material dependence of the cross-membrane ionic current, we coated a $\mathrm{SiO}_{2}$ layer of $50 \mathrm{~nm}$ thickness on the non-doped Si by chemical vapor deposition. Surprisingly, it caused only a marginal change in the resistive pulse waveforms from those found in the non-doped Si chips (Fig. 2e), whereas the open pore current became lower to a level comparable to that in the nanopore on doped Si (Fig. 2a; see also Fig. S2†). Similar effects have been observed by Lee and co-workers, ${ }^{13}$ wherein they attributed them to the suppression of electrochemical reactions at the Si surface. However, whether the results in Fig. 2 can also be explained by the surface effect needs further verification.

\section{Leakage current via unintentionally formed pinholes}

We thus explored the physical origin of the peculiar open pore conductance (Fig. 3) by measuring the $I_{\text {ion }}$ versus bias voltage $V_{\mathrm{b}}$ characteristics. We investigated the salt concentration $\left(c_{\text {ion }}\right)$ dependence of the open pore ionic conductance $G_{\text {open }}$. By diluting 10× PBS with ultrapure water (Merck Millipore), we prepared an electrolyte solution consisting mostly of $\mathrm{Na}^{+}$and $\mathrm{Cl}^{-}$ions with a vast range of $c_{\text {ion }}$ from $1.4 \mathrm{M}$ down to $1.4 \times 10^{-6}$ $\mathrm{M}$ (Fig. 3a and b). The results for $1.2 \mu \mathrm{m}$ sized $\mathrm{Si}_{3} \mathrm{~N}_{4}$ pores showed a linear decrease in $G_{\text {pore }}$ with decreasing salt concentration at $c_{\text {ion }}>10^{-3} \mathrm{M}$ irrespective of the substrate materials manifesting the drift current characteristic of $I_{\text {ion }}$ derived from a

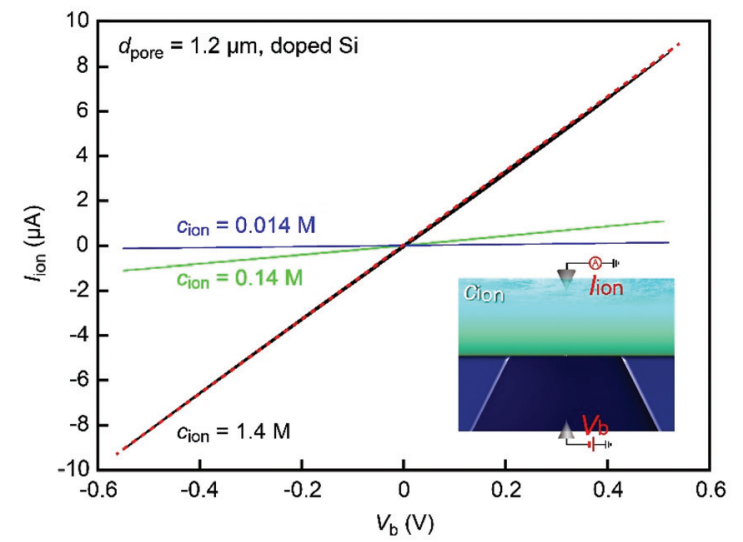

C

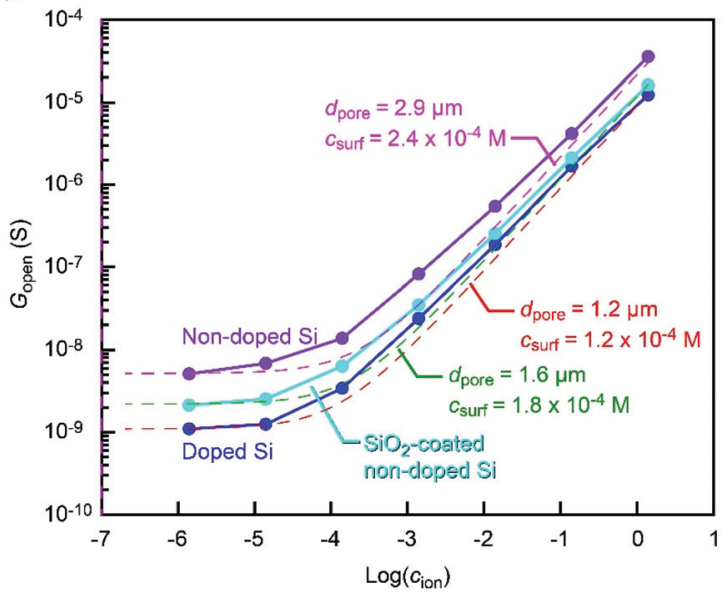

b

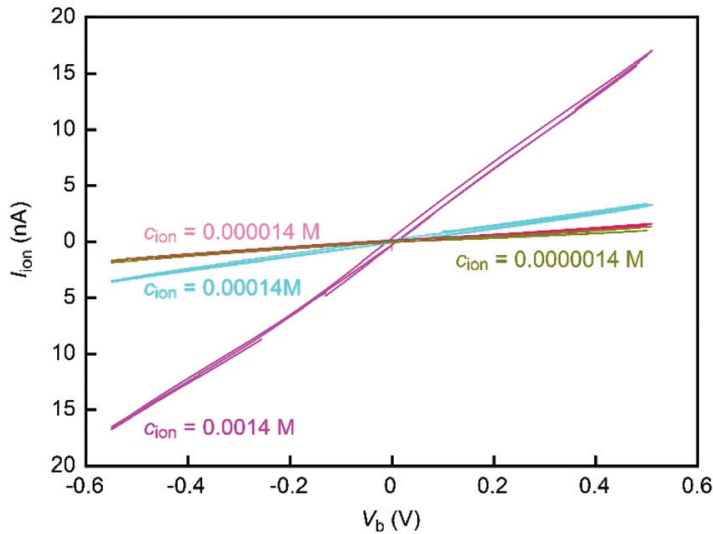

d

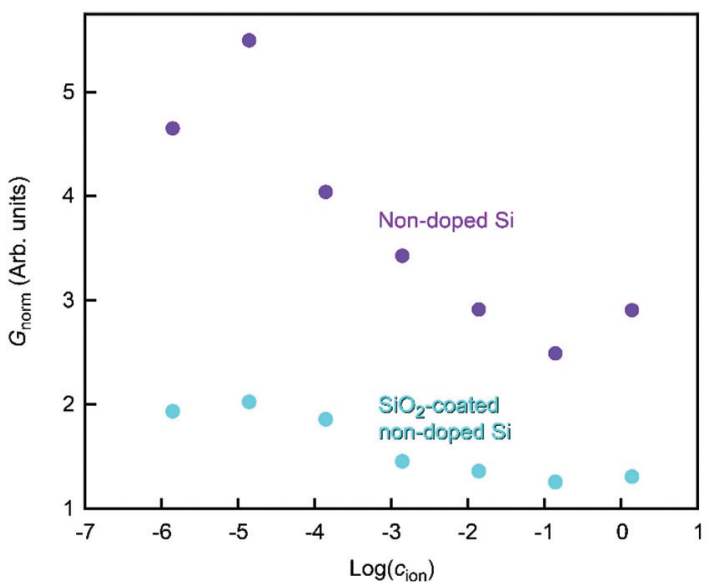

Fig. 3 Substrate material dependence of the open pore ionic conductance. (a and b) $l_{\text {ion }}$ versus applied voltage $V_{\mathrm{b}}$ characteristics measured in PBS buffer of various salt concentrations $c_{\text {ion }}$ of high (a) and low (b) ranges. Red dashed line in (a) is a linear fit to the curve at $c_{\text {ion }}=1.4 \mathrm{M}$, from which we deduced the open pore conductance $G_{\text {open. }}$ (c) Salt concentration dependence of $G_{\text {open. }}$. The dashed line is a fit presuming contributions of the ions

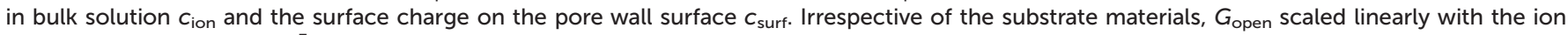
concentration at $c_{\text {ion }}>10^{-3} \mathrm{M}$. On the other hand, $G_{\text {open }}$ tended to level off at lower $c_{\text {ion, }}$ suggesting a pronounced influence of the pore wall surface charges. (d) $G_{\text {open }}$ normalized, $G_{\text {norm }}$, by that of doped Si plotted with respect to $\log \left(c_{\text {ion }}\right)$. 
the electric field-driven ion transport through the conduits (Fig. 3c). ${ }^{24}$ In contrast, $G_{\text {pore }}$ tended to level off at a certain level when further lowering $c_{\text {ion }}$. This electrolyte-independent ionic current can be attributed to predominant contributions of counterions at the pore wall surface. ${ }^{25}$

Meanwhile, it is also noticeable that the conductance remains in the order (doped $\mathrm{Si})<\left(\mathrm{SiO}_{2}\right.$-coated non-doped $\left.\mathrm{Si}\right)<$ (non-doped $\mathrm{Si}$ ) over the entire $c_{\text {ion }}$ range examined (Fig. 3c), which is consistent with that shown in Fig. 2. To characterize the relative difference, we normalized $G_{\text {open }}$ by that of nondoped Si. The thus obtained $G_{\text {norm }}$ demonstrated that while the conductance ratio remains almost constant at $c_{\text {ion }}>10^{-2}$ $\mathrm{M}$, the discrepancy becomes more pronounced at lower ion concentrations until it saturates at $c_{\text {ion }}<10^{-4} \mathrm{M}$ (Fig. 3d). This rather complicated behavior suggests a difference in the size of the ion-carrying channel in the $\mathrm{Si}_{3} \mathrm{~N}_{4}$ membrane. For example, $G_{\text {norm }}$ at the high $c_{\text {ion }}$ regime indicates a larger amount of drift current suggestive of a larger space for the ions to traverse the membranes on the non-doped Si substrates. Although no conspicuous difference in $d_{\text {pore }}$ was found under a scanning electron microscope together with any signs of additional channels in the membranes, it implies that some tiny pinholes were inadvertently created in the ultrathin membranes that mutually contributed to raising $G_{\text {open }}$ for the cases of the nondoped substrates. As for the comparatively lower conductance in the nanopore on $\mathrm{SiO}_{2}$-coated $\mathrm{Si}$, it can be due to the filling of the pin holes by the deposited $\mathrm{SiO}_{2}$ layer.

More quantitatively, the $G_{\text {open }}-c_{\text {ion }}$ characteristics can be compared to the aforementioned theoretical model of $G_{\text {pore }}=$ $\sigma d_{\text {pore }}$, wherein we ignored the negligibly small resistance inside the pore compared to the access resistance in the present low thickness-to-diameter aspect-ratio pores. In the equation, the solution conductivity $\sigma$ can be described as $\alpha c_{\text {net }}$, where $\alpha$ is a constant and $c_{\text {net }}$ is the concentration of ions relevant to the ionic current. Considering the role of the wall surface charges on $G_{\text {open }}, c_{\text {net }}$ is further approximated to be $c_{\text {net }}=c_{\text {ion }}+c_{\text {surf }}$, where $c_{\text {surf }}$ is the concentration of the surface charge-induced mobile ions. Indeed, Fig. $3 c$ can be fitted by different $d_{\text {pore }}$ values from $1.2 \mu \mathrm{m}$ of the doped Si to $2.9 \mu \mathrm{m}$ of the non-doped $\mathrm{Si}$ with similar $c_{\text {surf }}$ in the range of $10^{-4}$ $M$. These results corroborate a variation in the net size of ion carrying pores in the membrane. We emphasize that while there seem to be additional small channels, the pore to detect nanoparticles had a size as defined in the nanofabrication processes as confirmed by electron microscopy observations that ensures no notable difference in the resistive pulse patterns among the Si substrates used.

\section{Blunted resistive pulses in a high-resistance pore}

It therefore remains unclear why the resistive pulses became larger in the non-doped $\mathrm{Si}$ chips even when taking into account the experimental errors in the diameter of pores (Fig. S1 $\dagger$ ) as well as distributions in the size of nanoparticles $(<5 \%)$. In this context, it is noticeable that the substrate effects on ionic blockades were found to be more pronounced in resistive channels as described below. The ionic current flowing through a pore of $d_{\text {pore }}=1.2 \mu \mathrm{m}$ on doped Si in $0.1 \times$ PBS was found to be $15 \mathrm{nA}$ at $0.1 \mathrm{~V}$ (Fig. $4 \mathrm{a}$ and b). The corresponding $R_{\text {pore }}$ of $6.7 \mathrm{M} \Omega$ was about a factor of 4 larger than that in the $300 \mathrm{~nm}$ sized nanopore in $1 \times$ PBS (Fig. 2b). In this relatively high-resistance pore, the resistive pulses (obtained for $780 \mathrm{~nm}$ sized carboxylated polystyrene beads) showed a marked difference in their height when changing the support material from doped Si to non-doped Si (Fig. 4c-e).

A possible explanation for the peculiar substrate dependence of ionic current blockade is signal retardation due to the charging of capacitance in the nanopore system, ${ }^{18}$ which is described by the time constant $\tau=R_{\text {pore }} C_{\text {net }}$, where $C_{\text {net }}$ is the net capacitance of the whole chip. Indeed, the spike-like signals in the $1.2 \mu \mathrm{m}$ sized pore can be fitted with an exponential decay function $I_{\text {ion }}=I_{0} \exp \left(-t / \tau_{\text {decay }}\right)$ with a longer time constant $\tau_{\text {decay }}(633 \mu \mathrm{s})$ for doped Si compared to the nondoped Si counterpart $(288 \mu \mathrm{s})$ for the case of $1.2 \mu \mathrm{m}$ sized pores (Fig. 5a-c, see also Fig. S3†). The tendency was qualitatively the same in the $300 \mathrm{~nm}$ sized nanopores due in part to the lower $R_{\text {pore }}$ (Fig. 5d, see also Fig. S4†). This strongly suggests a prominent role of substrate-material-derived RC effects in the ionic current measurements: the resistive pulses in a pore on doped $\mathrm{Si}$ (non-doped $\mathrm{Si}$ ) were significantly (less) retarded by the long (short) charging time at the capacitive components of the Si chips.

\section{Substrate-capacitance-derived signal retardation in a nanopore}

How exactly did the substrate contribute to the $I_{\text {ion }}$ response to single-nanoparticle translocation then? In the description of time constant, the first term $R_{\text {pore }}$ is solely determined by buffer resistivity and nanopore geometry, both of which were arranged to be the same in the experiments (except the anomalous leakage-like current in non-doped Si chips). This is in fact evident in the $I_{\text {ion }}$ traces (Fig. 2a and $4 \mathrm{~b}$ ) showing a similar open pore current through the nanoscale conduit in two different substrates of doped $\mathrm{Si}$ and $\mathrm{SiO}_{2}$-coated non-doped $\mathrm{Si}$.

With $R_{\text {pore }}$ being not so different, the distinct difference in the resistive pulse waveforms should be attributed to $C_{\text {net }}$. Usually, the nanopore structure is modeled as a parallel circuit of $R_{\text {pore }}$ and the cross-membrane capacitance $C_{\text {net }}$ (Fig. 6). In the present device architecture, $C_{\text {net }}$ can be modeled as serialconnected capacitors of $C_{\mathrm{EDL}} / 2, C_{\mathrm{Si}_{3} \mathrm{~N}_{4}}$, and $C_{\mathrm{Si}}$ denoting the capacitance of the electric double layers, the $50 \mathrm{~nm}$ thick $\mathrm{Si}_{3} \mathrm{~N}_{4}$ regions, and the $525 \mu \mathrm{m}$ thick Si support, respectively. Among the components, $C_{\mathrm{EDL}}$ is appreciably larger than the others, ${ }^{26}$ and thus less important when connected in series. Whether the Si layer affects the ion blockade current therefore depends on the relative size of $C_{\mathrm{Si}_{3} \mathrm{~N}_{4}}$, and $C_{\mathrm{Si}}$. Based on the fact that the change in the composition of Si led to a pronounced effect on the resistive pulse patterns, $C_{\mathrm{Si}}$ was anticipated to be smaller or at least comparable to $C_{\mathrm{Si}_{3} \mathrm{~N}_{4}}$. Indeed, from bulk physical properties, $C_{\mathrm{Si}_{3} \mathrm{~N}_{4}}$ and $C_{\mathrm{nSi}}$ of non-doped Si per area are estimated to be $65 \mathrm{nF} \mathrm{cm}^{-2}$ and $0.2 \mathrm{nF} \mathrm{cm}{ }^{-2}$, reflecting the two orders of magnitude difference in their thickness together with a minor influence of the relative permittivity of 11.7 and 7.3 for intrinsic Si and $\mathrm{Si}_{3} \mathrm{~N}_{4}$, respectively. $C_{\text {net }}$ is then deduced 
a

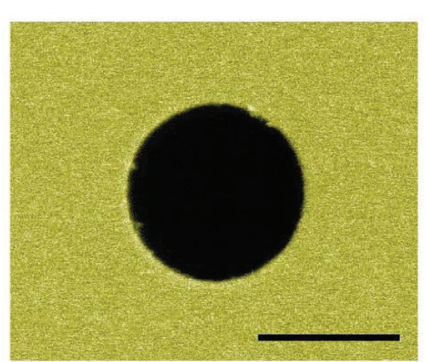

c

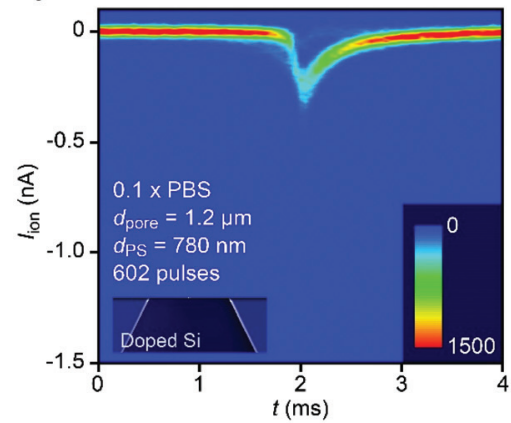

b

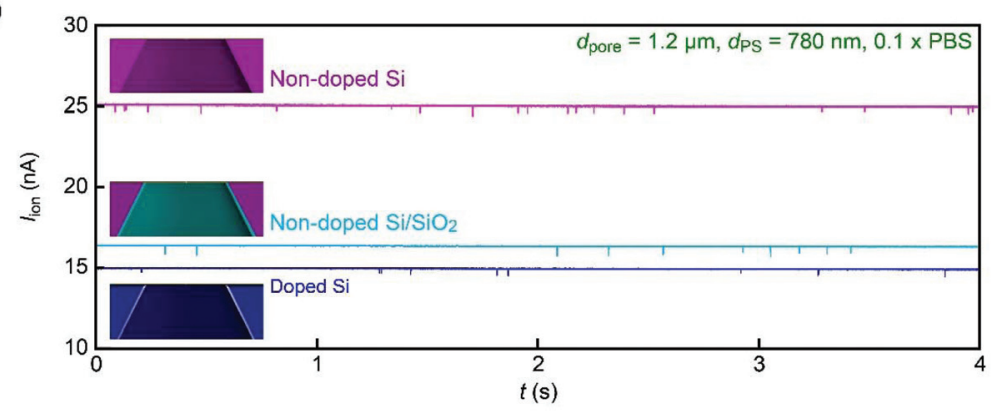

d

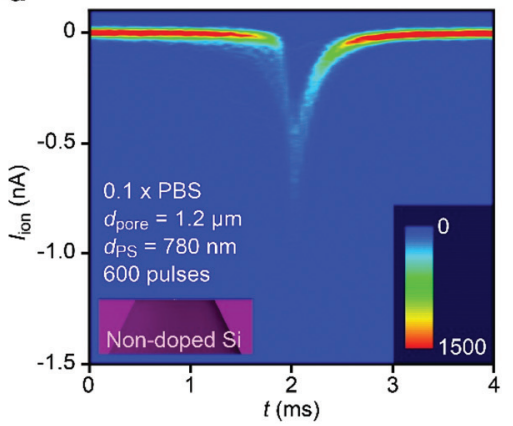

e

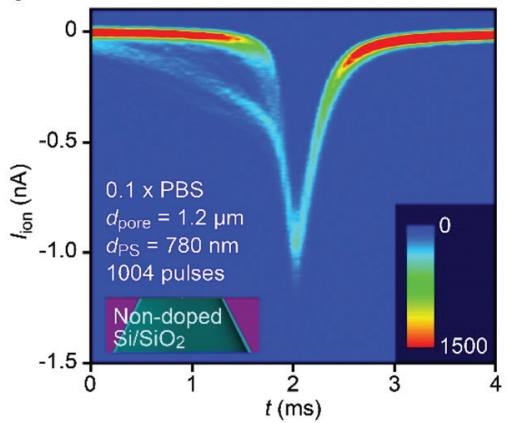

Fig. 4 Resistive pulse analysis under low ionic conductance conditions. (a) False-colored scanning electron micrograph of a micropore of $d_{\text {pore }}=$ $1.2 \mu \mathrm{m}$ in a $50 \mathrm{~nm}$ thick $\mathrm{Si}_{3} \mathrm{~N}_{4}$ membrane. Scale bar denotes $1 \mu \mathrm{m}$. (b) Typical ionic current curves obtained with the $1.2 \mu \mathrm{m}$ sized pore supported on a doped $\mathrm{Si}$ (blue), non-doped $\mathrm{Si}$ (purple), and $\mathrm{SiO}_{2}$-coated non-doped $\mathrm{Si}$ (skyblue). (c-e) Two-dimensional histograms showing a number of resistive pulses obtained with the $1.2 \mu \mathrm{m}$ sized pore supported on a doped $\mathrm{Si}$ (c), non-doped Si (d), and $\mathrm{SiO}_{2}$-coated non-doped $\mathrm{Si}$ (e). The open pore current is offset to zero.

a

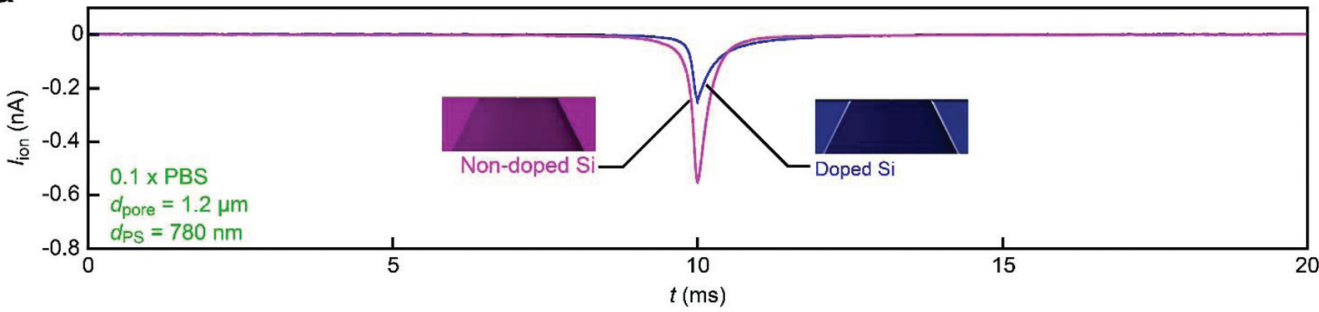

b

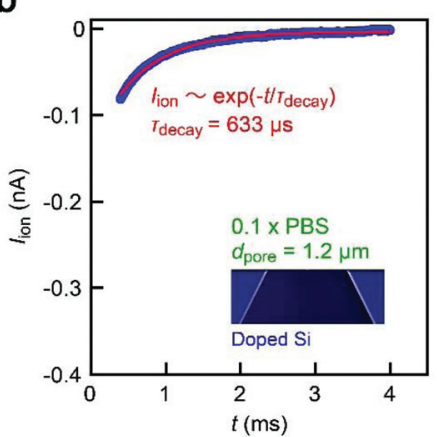

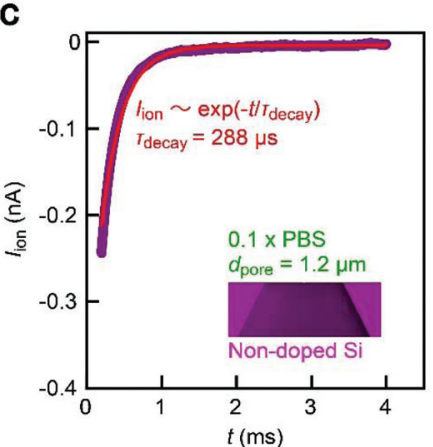

d

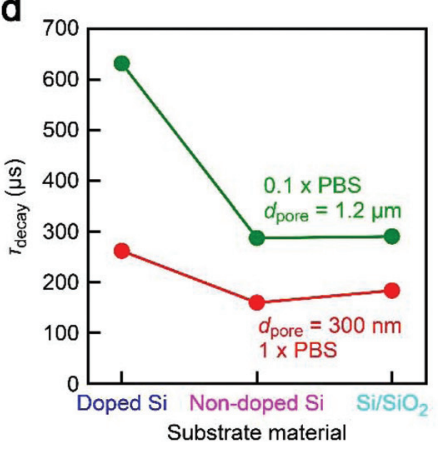

Fig. 5 Roles of substrate materials in the temporal response of the ionic current. (a) Average resistive pulses obtained with the $1.2 \mu \mathrm{m}$ sized pore for $780 \mathrm{~nm}$ sized polystyrene nanoparticles in 0.1x PBS. The open pore current is offset to zero. The pulse became significantly blunt in the nanopore on doped Si than that on non-doped Si. (b and c) lonic current decay at the resistive pulse tails for the $1.2 \mu \mathrm{m}$ sized pore on doped (b) and nondoped $\mathrm{Si}$ (c). The red curves are exponential fitting that provides the decay constant $\tau_{\text {decay. }}$ (d) Substrate material dependence of $\tau_{\text {decay. }}$. 

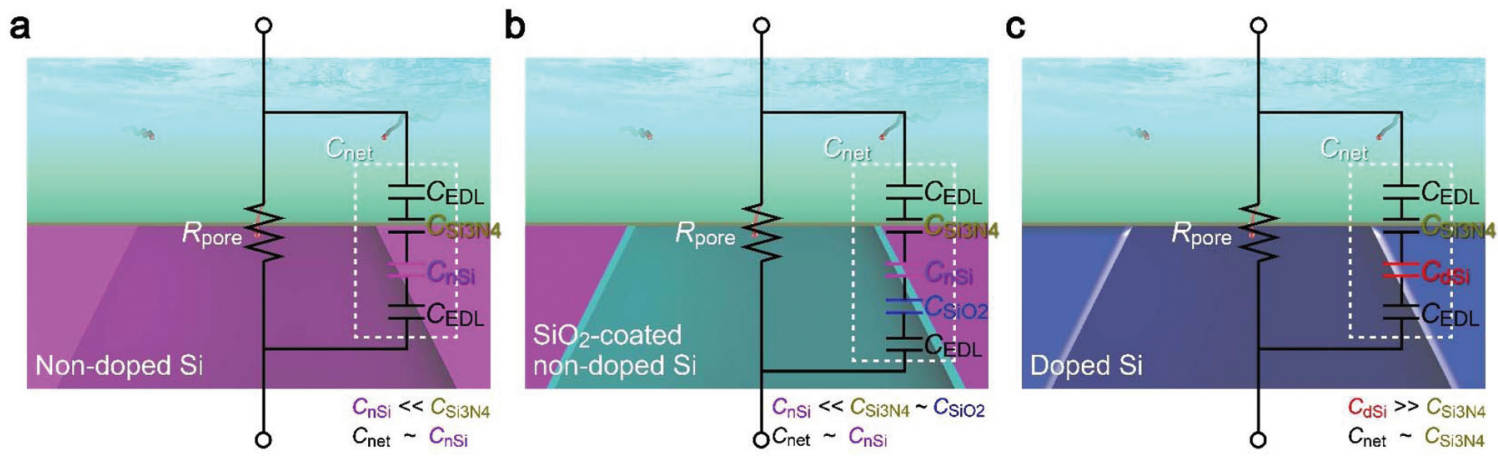

Fig. 6 Capacitance of nanopore chips. (a-c) Equivalent circuits of a nanopore on non-doped Si (a), $\mathrm{SiO}_{2}$-coated non-doped Si (b), and doped Si (c).

as $C_{\text {net }}=C_{\mathrm{Si}_{3} \mathrm{~N}_{4}} C_{\mathrm{nSi}} /\left(C_{\mathrm{Si}_{3} \mathrm{~N}_{4}}+C_{\mathrm{nSi}}\right) \sim C_{\mathrm{nSi}}$ for the intrinsic $\mathrm{Si}$ (Fig. 6a).

The discussion is the same for the case where non-doped $\mathrm{Si}$ is covered with $50 \mathrm{~nm}$ thick $\mathrm{SiO}_{2}$ as its capacitance is only comparable to that of the $\mathrm{Si}_{3} \mathrm{~N}_{4}$ layer, and hence contributes little to $C_{\text {net }}$ due to the significantly smaller $C_{\mathrm{nSi}}$ connected in series (Fig. 6b). In contrast, the high conductivity of doped $\mathrm{Si}$ suggests a huge dielectric constant ${ }^{27,28}$ that makes its capacitance $C_{\mathrm{dSi}}$ to be excessively larger than $C_{\mathrm{Si}_{3} \mathrm{~N}_{4}}$, and hence $C_{\text {net }} \sim$ $C_{\mathrm{Si}_{3} \mathrm{~N}_{4}}$. As a consequence, the net capacitance became much larger for the nanopore on doped $\mathrm{Si}$, thereby rendering inadequate temporal resolution of the ionic current measurements for detecting the fast ion blockade events by the singlenanoparticle translocation (Fig. 6c). In fact, when assuming the real translocation time to be $287 \mu \mathrm{s}$, the pulses should be detected as $288 \mu$ s and $633 \mu$ s wide signals on non-doped and doped Si considering the RC delay with the relative difference in the specific capacitance, which is in fair agreement with the experimental results (Fig. 5). The overall findings can be used to achieve a faster temporal response of the ionic current and better signal-to-noise ratio in resistive pulse analyses using Sibased solid-state nanopores.

\section{Signal retardation in smaller nanopores}

It is interesting to estimate the RC effect in smaller nanopores. For this, we calculated $R_{\text {net }}$ using an access resistance model ${ }^{29}$ as $R_{\text {net }}=R_{\text {pore }}+R_{\text {acc }}$, where $R_{\text {pore }}=R_{\text {pore }}=4 \rho L / \pi d_{\text {pore }}^{2}$ and $R_{\text {acc }}=\rho / d_{\text {pore }}$ are, respectively, the resistance inside and outside the pore of diameter $d_{\text {pore }}$ and depth $t_{\text {pore }}=50 \mathrm{~nm}$. The solution resistivity $\rho$ in the equation was tentatively assumed to be $0.8 \Omega \mathrm{m}$ for $1 \times$ PBS. Then, we deduced the time constant for the cases of $C_{\text {net }}=95 \mathrm{pF}$ and $0.3 \mathrm{pF}$ corresponding to the properties of a $50 \mathrm{~nm}$ thick $\mathrm{Si}_{3} \mathrm{~N}_{4}$ membrane on doped and non-doped $500 \mu \mathrm{m}$ thick Si substrates, respectively. The thus obtained $\tau_{\text {delay }}-d_{\text {pore }}$ dependence (Fig. 7) suggested a significant influence of $R_{\text {net }}$ on nanopores smaller than $100 \mathrm{~nm}$ under the conditions assumed. The detrimental influence is expected to become more significant when using a thinner membrane, which is a prerequisite for sensing ultrafine particles and molecules such as proteins ${ }^{30}$ and polynucleotides. ${ }^{31}$ This predicts the importance to devote additional efforts to

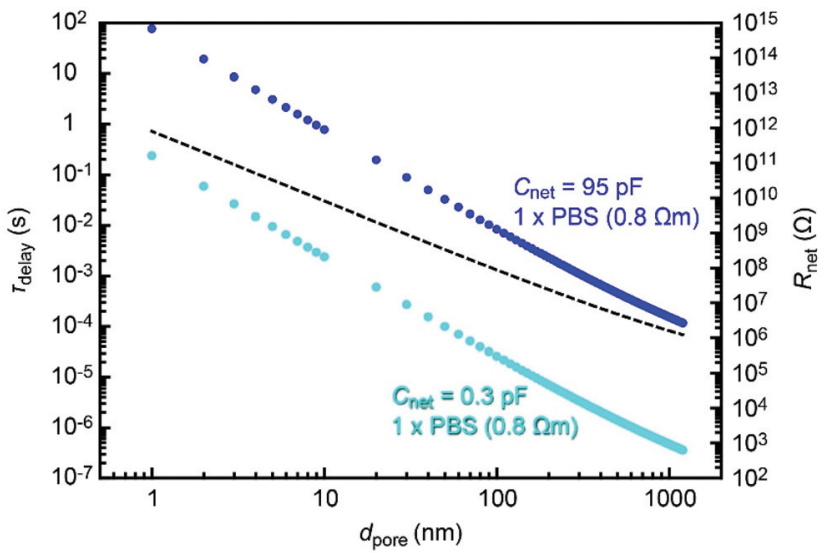

Fig. 7 Time constant in smaller nanopores. Blue and sky blue plots are $\tau_{\text {delay }}$ obtained for a pore of diameter $d_{\text {pore }}$ in a $50 \mathrm{~nm}$ thick $\mathrm{Si}_{3} \mathrm{~N}_{4} \mathrm{mem}$ brane supported on doped and non-doped $\mathrm{Si}$, respectively, in $1 \times$ PBS. The dashed line describes the $d_{\text {pore }}$ dependence of the pore resistance $R_{\text {net }}$

lower the capacitance by shrinking the water-touching area of thin dielectrics ${ }^{17}$ and also to decrease the net resistance by using an electrolyte solution with a higher ion concentration.

\section{Dielectric breakdown as a possible cause of pinhole generation}

The variation in nanopore chip capacitance infers dielectric breakdown in a $\mathrm{Si}_{3} \mathrm{~N}_{4}$ membrane as a cause of the anomalous cross-pore ionic current characteristics on non-doped Si. Yanagi et al. ${ }^{17}$ reported that electric charge imbalance between the two sides of a thin $\mathrm{Si}_{3} \mathrm{~N}_{4}$ membrane would trigger a local fracture at intrinsic defects in the material in a manner akin to dielectric breakdown. The phenomenon was found to occur more easily in devices with lower capacitance due to the higher voltage induced upon injecting an electrolyte solution to a membrane. ${ }^{32}$ The resulting leakage current through the created pinholes became larger with decreasing capacitance. ${ }^{32}$ This would be a plausible explanation for the present results: the lower (higher) capacitance of the non-doped (doped) $\mathrm{Si}$ chips tends to trigger (hinder) dielectric breakdown yielding the larger (negligible) leakage-like ionic current through the 
membrane; meanwhile, $\mathrm{SiO}_{2}$ deposition on non-doped Si contributed to slightly enlarge the net capacitance, thereby partially preventing electrical breakdown. The overall findings consistently indicate the importance of the substrate material not only for achieving a high temporal resolution of nanopore sensing but also for preventing unintentional formations of nanoholes in a membrane.

\section{Conclusions}

We systematically evaluated the influence of $\mathrm{Si}$ substrate materials on the performance of $\mathrm{Si}_{3} \mathrm{~N}_{4}$ nanopore single-particle detectors. Although the only difference in the silicon substrate materials is the amount of impurities, and despite the fact that the substrate is located far away from the pore and so hardly imaginable to influence the cross-pore ion transport, the results demonstrated a pronounced influence on the ionic current sensitivity to single-nanoparticle translocation. Specifically, when a nanopore on a doped Si wafer was used, the relatively small capacitance at the thick silicon provided a short RC time constant with fast response of $I_{\text {ion }}$ to the fast electrophoretic translocation of nanoparticles through the nanoscale conduit. In the case of doped $\mathrm{Si}$, in contrast, its huge capacitance eventually raised the net capacitance of the device chip compared to that on the non-doped Si, thereby causing significant retardation of resistive pulse signals. The present results prove the usefulness of highly insulating silicon as a substrate material for attaining better performance of solid-state nanopore sensors.

\section{Methods}

\section{Chemicals and materials}

$\mathrm{Si}_{3} \mathrm{~N}_{4}$-coated silicon wafers were purchased from Electronics and Materials. Carboxylated polystyrene nanoparticles were purchased from Polyscience and used without any filtering. Phosphate buffered saline (PBS) was purchased from Wako. Ultra-pure water with a resistivity of $18.2 \mathrm{M} \Omega \mathrm{cm}$ was obtained using a Milli-Q Millipore system (Merck Millipore). Electron beam resist ZEP520A was purchased from Zeon.

\section{Solid-state nanopore fabrications}

Solid-state nanopores were fabricated as follows. A $525 \mu \mathrm{m}$ thick silicon wafer with $50 \mathrm{~nm}$ thick $\mathrm{Si}_{3} \mathrm{~N}_{4}$ layers grown on both sides via low-pressure chemical vapor deposition was diced into $30 \mathrm{~mm} \times 30 \mathrm{~mm}$ chips using a dicer. The Si layer was either non-doped (conductivity $<1000 \Omega \mathrm{cm}$ ) or phosphordoped (conductivity $=1-20 \Omega \mathrm{cm}$ ). $1 \mathrm{~mm} \times 1 \mathrm{~mm}$ area of $\mathrm{Si}_{3} \mathrm{~N}_{4}$ was removed by reactive ion etching using $\mathrm{CF}_{4}$ gas. The exposed $\mathrm{Si}$ was then deep-etched in $\mathrm{KOH}$ solution heated to 80 degrees celsius. As a result, a $50 \mathrm{~nm}$ thick $\mathrm{Si}_{3} \mathrm{~N}_{4}$ membrane of approximately $100 \mu \mathrm{m} \times 100 \mu \mathrm{m}$ square dimension was formed at the bottom of the pyramidal-shaped trench. On the membrane, we spin-coated an electron beam resist (ZEP520A, Zeon) for electron-beam-drawing a nanopore. In the case of the nondoped Si substrate, we added a conductive spacer (ESPACER, Showa Denko) to avoid charge-up. A circle of diameter $d_{\text {pore }}$ was then rendered by a standard electron-beam lithography method in a membrane. After development, the remaining resist was used as a mask to drill a nanopore by isotropically etching the surface by reactive ion etching (etchant gas = $\mathrm{CHF}_{3}$ ). Then, the residual resist was dissolved in $N, N$-dimethylformamide followed by rinsing in ethanol and acetone. Before the measurements, we sealed the nanopore chip from both sides with two polymer blocks made of polydimethylsiloxane (PDMS). This was done by first activating the surface with oxygen plasma followed by attachment to the chip surface for eternal bonding. In the PDMS blocks, there was a microchannel to inject liquid into the nanopore.

\section{Ionic current measurements}

Resistive pulse analyses of single nanoparticles were implemented by measuring the ionic current $I_{\text {ion }}$ through a nanopore of diameter $d_{\text {pore }}$ sculpted in a $50 \mathrm{~nm}$ thick $\mathrm{Si}_{3} \mathrm{~N}_{4}$ membrane under the applied dc voltage of $0.1 \mathrm{~V}$ using two $\mathrm{Ag} /$ $\mathrm{AgCl}$ electrodes. One side of the membrane was filled with a dilute dispersion solution of carboxylated polystyrene beads of diameter $d_{\mathrm{PS}}$ in PBS, while the other side was immersed in PBS only. The voltage created a huge electric field at the nanopore that served to electrophoretically draw the negatively charged nanobeads into the channel. As a result, $I_{\text {ion }}$ tended to drop rapidly as each nanoparticle passed through the conduit due to the temporal block of the in-pore ion transport. The time-course change in the ionic current was recorded at a sampling rate of $1 \mathrm{MHz}$ without any filter by using a homebuilt current amplifier backed by a fast digitizer (NI-5922, National Instruments) using a LabVIEW program.

\section{Resistive pulse extraction}

The moving open pore current was offset to zero by subtracting the linearly fitted base current in every 0.5 seconds of the $I_{\text {ion }}$ versus $t$ data. Resistive pulses were then obtained by searching for local minima below a threshold level followed by extraction of 0.005 seconds of data before and after each pulse apex.

\section{Conflicts of interest}

There are no conflicts to declare.

\section{Acknowledgements}

A part of this work was supported by the ImPACT Program of the Council for Science, Technology, and Innovation (Cabinet Office, Government of Japan), the "Nanotechnology Platform Project (Nanotechnology Open Facilities in Osaka University)" of the Ministry of Education, Culture, Sports, Science and Technology, Japan [No: F-12-OS-0016], and the Japan Society 
for the Promotion of Science (JSPS) KAKENHI Grant Number $18 \mathrm{H} 01846$.

\section{Notes and references}

1 J. E. Reiner, A. Balijepalli, J. W. F. Robertson, J. Campbell, J. Suehle and J. J. Kasianowicz, Chem. Rev., 2012, 112, 6431-6451.

2 C. Dekker, Nat. Nanotechnol., 2007, 2, 209-215.

3 L. Luo, S. R. German, W.-J. Lan, D. A. Holden, T. L. Mega and H. S. White, Annu. Rev. Anal. Chem., 2014, 7, 513-535.

4 S. Howorka and Z. Siwy, Chem. Soc. Rev., 2009, 38, 2360-2384.

5 D. G. Haywood, A. Saha-Shah, L. A. Baker and S. C. Jacobson, Anal. Chem., 2011, 6, 615-624.

6 W. H. Coulter, US patent, 2656508, 1953.

7 C. Y. Kong and M. Muthukumar, J. Chem. Phys., 2004, 120, 3460.

8 H. Yasaki, T. Yasui, T. Yanagida, N. Kaji, M. Kanai, K. Nagashima, T. Kawai and Y. Baba, J. Am. Chem. Soc., 2017, 139, 14137-14142.

9 E. C. Yusko, B. R. Bruhn, O. M. Eggenberger, J. Houghtaling, R. C. Rollings, N. C. Walsh, S. N. Nandivada, M. Pindrus, A. R. Hall, D. Sept, J. Li, D. S. Kalonia and M. Mayer, Nat. Nanotechnol., 2017, 12, 360-368.

10 M. Tsutsui, T. Yoshida, K. Yokota, H. Yasaki, T. Yasui, A. Arima, W. Tonomura, K. Nagashima, T. Yanagida, N. Kaji, M. Taniguchi, T. Washio, Y. Baba and T. Kawai, Sci. Rep., 2017, 7, 17371.

11 J. W. F. Robertson, C. G. Rodrigues, V. M. Stanford, K. A. Rubinson, O. V. Krasilnikov and J. J. Kasianowicz, Proc. Natl. Acad. Sci. U. S. A., 2007, 104, 8207-8211.

12 N. Arjmandi, W. Van Roy, L. Lagae and G. Borghs, Anal. Chem., 2012, 84, 8490-8496.

13 M.-H. Lee, J.-H. Lee, H.-M. Kim, Y.-R. Kim, T.-J. Jeon, Y. E. Pak and K.-B. Kim, Microfluid. Nanofluid., 2014, 16, 123-130.

14 A. Arima, A. H. Harlisa, T. Yoshida, M. Tsutsui, M. Tanaka, K. Yokota, W. Tonomura, J. Yasuda, M. Taniguchi, T. Washio, M. Okochi and T. Kawai, J. Am. Chem. Soc., 2018, 140, 16834-16841.
15 V. Tabard-Cossa, D. Trivedi, M. Wiggin, N. N. Jetha and A. Marziali, Nanotechnology, 2007, 18, 305505.

16 S. Shekar, D. J. Niedzwiecki, C.-C. Chien, P. Ong, D. A. Fleischer, J. Lin, J. K. Rosenstein, M. Drndic and K. L. Shepard, Nano Lett., 2016, 16, 4483-4489.

17 Y. Goto, I. Yanagi, K. Matsui, T. Yokoi and K.-I. Takeda, Sci. Rep., 2016, 6, 31324.

18 M. Tsutsui, K. Yokota, A. Arima, W. Tonomura, M. Taniguchi, T. Washio and T. Kawai, ACS Appl. Mater. Interfaces, 2018, 10, 34751-34757.

19 T. Jain, R. J. S. Guerrero, C. A. Aguilar and R. Karnik, Anal. Chem., 2013, 85, 3871-3878.

20 M.-H. Lee, A. Kumar, K.-B. Park, S.-Y. Cho, H.-M. Kim, M.-C. Lim, Y.-R. Kim and K.-B. Kim, Sci. Rep., 2014, 4, 7448.

21 S. W. Kowalczyk, A. Y. Grosberg, Y. Rabin and C. Dekker, Nanotechnology, 2011, 22, 315101.

22 M. Tsutsui, S. Hongo, Y. He, M. Taniguchi, N. Gemma and T. Kawai, ACS Nano, 2012, 6, 3499-3505.

23 M. Tsutsui, Y. He, K. Yokota, A. Arima, S. Hongo, M. Taniguchi, T. Washio and T. Kawai, ACS Nano, 2016, 10, 803-809.

24 W. Guan, R. Fan and M. A. Reed, Nat. Commun., 2011, 2, 506.

25 C. Lee, L. Joly, A. Siria, A.-L. Biance, R. Fulcrand and L. Bocquet, Nano Lett., 2012, 12, 4037-4044.

26 B. Skinner, M. S. Loth and B. I. Shklovskii, Phys. Rev. Lett., 2010, 104, 128302.

27 S. Dhar and A. H. Marshak, Solid-State Electron., 1985, 28, 763-766.

28 S. D. Ristic, Z. D. Prijic and S. Z. Mijalkovic, Phys. Status Solidi, 1995, 148, 575.

29 S. W. Kowalczyk, A. Y. Grosberg, Y. Rabin and C. Dekker, Nanotechnology, 2011, 22, 315101.

30 J. Wilson, L. Sloman, Z. He and A. Aksimentiev, Adv. Funct. Mater., 2016, 26, 4830-4838.

31 D. B. Wells, M. Belkin, J. Comer and A. Aksimentiev, Nano Lett., 2012, 12, 4117-4123.

32 K. Matsui, I. Yanagi and K.-I. Takeda, Sci. Rep., 2015, 5, 17819. 